\title{
Neural Representations of Courtship Song in the Drosophila Brain
}

\author{
Sina Tootoonian, ${ }^{1}$ Philip Coen, ${ }^{2}$ Risa Kawai, ${ }^{1}$ and Mala Murthy ${ }^{1,2}$ \\ ${ }^{1}$ Computation and Neural Systems Program and Division of Biology, California Institute of Technology, Pasadena, California 91125, and ${ }^{2}$ Princeton \\ Neuroscience Institute and Department of Molecular Biology, Princeton University, Princeton, New Jersey 08544
}

Acoustic communication in drosophilid flies is based on the production and perception of courtship songs, which facilitate mating. Despite decades of research on courtship songs and behavior in Drosophila, central auditory responses have remained uncharacterized. In this study, we report on intracellular recordings from central neurons that innervate the Drosophila antennal mechanosensory and motor center (AMMC), the first relay for auditory information in the fly brain. These neurons produce graded-potential (nonspiking) responses to sound; we compare recordings from AMMC neurons to extracellular recordings of the receptor neuron population [Johnston's organ neurons (JONs)]. We discover that, while steady-state response profiles for tonal and broadband stimuli are significantly transformed between the JON population in the antenna and AMMC neurons in the brain, transient responses to pulses present in natural stimuli (courtship song) are not. For pulse stimuli in particular, AMMC neurons simply low-pass filter the receptor population response, thus preserving low-frequency temporal features (such as the spacing of song pulses) for analysis by postsynaptic neurons. We also compare responses in two closely related Drosophila species, Drosophila melanogaster and Drosophila simulans, and find that pulse song responses are largely similar, despite differences in the spectral content of their songs. Our recordings inform how downstream circuits may read out behaviorally relevant information from central neurons in the AMMC.

\section{Introduction}

To select appropriate mates, animals must be able to tell conspecific from heterospecific and to judge fitness among conspecific suitors. Animals take advantage of several sensory channels to make these distinctions, but the use of acoustic cues is quite prevalent across the animal kingdom (Suga, 1989; Bass and McKibben, 2003; Kelley, 2004; Mason and Faure, 2004; Theunissen and Shaevitz, 2006). Processing behaviorally relevant acoustic signals typically involves neural tuning for conspecific sounds, and such tuning has been reported in animals as divergent as insects, birds, and primates (Bentley and Hoy, 1972; Braaten and Reynolds, 1999; Machens et al., 2005; Petkov et al., 2008). However, it is not known how auditory systems achieve their exquisite tuning for conspecific signals, nor how precisely auditory responses are

\footnotetext{
Received 0ct. 9, 2011; accepted Nov. 13, 2011

Author contributions: S.T. and M.M. designed research; P.C., R.K., and M.M. performed research; S.T., P.C., and M.M. analyzed data; M.M. wrote the paper.

This work was supported by NIH-NIDCD Grant R01-DC7652 (to Gilles Laurent), funding from the Max Planck Society and a Natural Sciences and Engineering Research Council of Canada PGS-M award (S.T.), and a Helen Hay Whitney postdoctoral fellowship and start-up funds from Princeton University (M.M.). M.M. is also funded through an NSF CAREER award, and through grants from the Human Frontiers Science Program (HFSP) and the Alfred P. Sloan Foundation. We are extremely grateful to Gilles Laurent for critical advice on and funding for this project, to members of the Laurent Laboratory for valuable feedback, to Mark Konishi for donating equipment for fly song recordings, and to Hidehiko Inagaki for help with anatomical characterization of the GAL4 lines used in this study. We also thank Azusa Kamikouchi and Joerg Albert for comments on this manuscript.

Correspondence should be addressed to Mala Murthy, Princeton Neuroscience Institute and Department of Molecular Biology, Princeton University, 328 Moffett, Washington Road, Princeton, NJ 08544. E-mail: mmurthy@princeton.edu.

S. Tootoonian's present address: Max Planck Institute for Brain Research, Frankfurt, Germany, 60528.

R. Kawai's present address: Biophysics Graduate Program, Harvard University, Boston, Massachusetts 02115.

DOI:10.1523/JNEUROSCI.5104-11.2012

Copyright $\odot 2012$ the authors $\quad 0270-6474 / 12 / 320787-12 \$ 15.00 / 0$
}

transformed from one layer of the nervous system to the next to accomplish such tuning.

To characterize auditory systems, synthetic stimuli (such as pure tones and broadband noise) have been extremely useful; these stimuli are simple to parameterize and control, and therefore responses are straightforward to quantify. However, recent studies using natural sounds have found that auditory neurons encode natural stimuli both differently from synthetic stimuli (Bar-Yosef et al., 2002; Woolley et al., 2006) and more efficiently (Rieke et al., 1995; Hsu et al., 2004; Smith and Lewicki, 2006). As a consequence, responses to synthetic stimuli are generally poor predictors of responses to natural stimuli (Rotman et al., 2001; David et al., 2004; Woolley et al., 2006). It is thought that the temporal structure present in natural stimuli forms the basis for this difference in tuning (Woolley et al., 2006), but the precise mechanisms remain unknown. We investigate this issue here using Drosophila, whose songs are, like those of other animals, highly temporally patterned. Studying auditory processing mechanisms in Drosophila provides a unique opportunity to discover general principles underlying how species-specific sounds are represented within the brain, given the simplicity of the fly nervous system and the wealth of genetic tools available to identify and study individual neurons.

In Drosophila, sound is first detected by the fly's antenna via vibrations of its feathery arista and associated third segment (Göpfert and Robert, 2001, 2002). Analogous to the mechanical coupling between the basilar membrane and auditory hair cells in vertebrates, the fly's auditory organ transduces mechanical deflections of the arista into receptor potentials within the $\sim 500$ Johnston's organ neurons (JONs) housed within the antenna 
(Todi et al., 2004; Albert et al., 2007; Nadrowski et al., 2011). JONs project along the antennal nerve into the brain, where they arborize in the antennal mechanosensory and motor center (AMMC) (Kamikouchi et al., 2006). Dependent on where their somata are located in the antenna, JONs project to one of roughly five zones in the AMMC - those that project to zones A and B are mostly sensitive to vibratory stimuli, such as courtship song, whereas those that innervate zones $\mathrm{C}$ and $\mathrm{E}$ respond more robustly to static stimuli, such as wind or gravity (Kamikouchi et al., 2009; Yorozu et al., 2009). In this study, we investigate auditory tuning among central neurons that innervate AMMC zones A and $\mathrm{B}$, and compare responses with those of the JON population.

Courtship song in drosophilid flies typically comprises two modes, pulse and sine. Pulse song, which is characterized by brief sound impulses separated by species-typical interpulse intervals, has been shown through behavioral experiments to be more salient for mate selection (Bennet-Clark and Ewing, 1969; von Schilcher, 1976). However, the majority of tuning curves formed for the Drosophila auditory receiver and coupled receptor neurons come from analyzing responses to synthetic stimuli, such as sinusoids and white noise (Göpfert and Robert, 2002; Göpfert et al., 2006; Effertz et al., 2011). These tuning curves describe responses at steady state, whereas responses to song pulses are likely to be transient. Here, we test the hypothesis that tuning for song pulses (natural sounds) in the central auditory system of Drosophila differs from steady-state tuning for synthetic stimuli. In addition, by making comparisons between the songs and auditory systems of two closely related and hybridizable Drosophila species, we offer insights into the neural basis for species-specific song recognition.

\section{Materials and Methods Fly lines}

Song recordings (from pairings of single virgin males with virgin females) and JON recordings (from virgin females) were collected from OrR [Drosophila melanogaster (D. mel)] and sim194 [Drosophila simulans (D. sim) collected from Winters, California (Dermitzakis et al., 2000)] strains. Whole-cell patch-clamp recordings were performed on virgin females of the following genotypes: AMMC-A1 [UAS-eGFP2x(II); c767-GAL4(III)]; giant fiber (GF) [c17-GAL4, UAS-CD8-GFP(III)]; AMMC-B1 [UAS-eGFP2x(II); 8-245-GAL4(III)]; AMMC-B2 [JO2(NP1046); UAS-eGFP2x(II)]. D. mel/D. sim hybrid females were generated by crossing UAS-eGFP2x; c767-GAL4 females to sim194 males. All flies were reared on standard cornmeal agar medium at constant temperature $\left(25^{\circ} \mathrm{C}\right)$ and constant relative humidity (65\%).

\section{Sound stimuli}

Our sound stimuli consisted of pure tones [17 4 s tones $(100-900 \mathrm{~Hz})$, each delivered at two intensities], white noise (two different $4 \mathrm{~s}$ variants of white noise, each delivered at three intensities), and fly song [10 $4 \mathrm{~s}$ song bouts from $D$. melanogaster (six stimuli) and D. simulans (four stimuli)]. For a few experiments, we used synthetic pulse stimuli, which were generated by cutting a single pulse from a $D$. mel song recording and creating pulse trains consisting of 10-20 pulses separated by interpulse intervals (IPIs) from 20 to 90 ms. Each 4 s auditory stimulus was spaced $20 \mathrm{~s}$ apart from the next stimulus. The intensity of each stimulus was computed using a $20 \mathrm{~ms}$ sliding fast Fourier transform (FFT) window (Hamming) applied to the signal recorded on a calibrated NR23158 (Knowles Electronics) pressure gradient microphone connected to a custom-built nonintegrating differential amplifier (1.6 Hz high-pass filter with $-3 \mathrm{~dB}$ roll-off; see below for more information on the frequency response of the microphone). The NR23158 microphone was calibrated in an anechoic chamber (Siapas Laboratory, Caltech, Pasadena, CA) at a distance of $2.3 \mathrm{~m}$ from the sound source (Fostex FX120), and according to the protocol provided in the study by Göpfert et al. (2006). The inten- sities of tonal and white-noise stimuli were then adjusted to be within the range from 0.1 to $3 \mathrm{~mm} / \mathrm{s}$; fly songs were played back at the intensity they were recorded, which fell roughly within this range.

\section{Sound delivery system and calibration}

Our sound delivery system consisted of the following: (1) M-Audio Audiophile 2496 sound card, (2) Samson S-phone four-channel headphone amplifier, (3) KOSS Sparkplug headphones (16 $\Omega$ impedance; sensitivity, $112 \mathrm{~dB}$ SPL/1 mW), and (4) $50 \mathrm{~cm}$ Nalgene 489 rigid polyethylene tubing [inner diameter (i.d.), 3/16 inch; outer diameter (o.d.), 5/16 inch] coupled to $5 \mathrm{~cm}$ of thinner tubing (i.d., 1/16 inch; o.d., 3/16 inch). We estimated the transfer function of the sound delivery system by playing 50 trials of a white-noise stimulus (bandpass filtered from 80 to $1000 \mathrm{~Hz}$ and delivered at a range of intensities) and recording the output on a Brüel \& Kjær 0.5 inch free-field condenser microphone (4133) in a custom-built soundproof box. We chose this microphone because its frequency response characteristics are flat $( \pm 2 \mathrm{~dB})$ within the lowfrequency range we tested. The transfer function between input $(x)$ and output $(y)$ was estimated using the quotient of the cross power spectral density (Pyx) of $x$ and $y$ and the power spectral density (Pxx) of $x$ (tfestimate function in MATLAB). We then compensated for distortions introduced by the headphone speaker and coupling tube by convolving our stimuli with the inverse transfer function filter before playing the sound through the system [our method for sound calibration is conceptually similar to that used in the studies by Göpfert and Robert (2003) and Wightman and Kistler (1989)]. Without compensation, the output of the system amplifies low frequencies $(100-300 \mathrm{~Hz})$ and attenuates higher frequencies $(300-1000 \mathrm{~Hz})$. After compensation, the output of the system is a near-perfect match with the input (see Fig. 1). The sound tube was placed roughly $2 \mathrm{~mm}$ from the fly's right antenna, and at a $45^{\circ}$ angle to the substrate [to mimic male singing (Bennet-Clark, 1971)]. The tube creates a planar sound wave, and we empirically determined that the response of the pressure gradient microphone, placed $2 \mathrm{~mm}$ from the end of the tube, increased linearly with frequency [that is, we observed no flattening of the frequency response of the microphone at short distances from the end of the tube, consistent with the study by Werner (2008)]. We compensated for the frequency response of microphone post hoc by dividing FFT voltages by their respective frequencies.

\section{Local field potential recordings from the Johnston's organ}

Recordings were made using a Brownlee Precision 440 differential amplifier (gain, 10,000) and by inserting a recording tungsten electrode (18 M $\Omega$ impedance) into the gap between antennal segments a1 and a 2 of the fly's right antenna and a reference tungsten electrode ( $7 \mathrm{M} \Omega$ impedance) into the eye [similar to the study by Eberl et al. (2000)]. Responses were bandpass filtered between $75 \mathrm{~Hz}$ and $10 \mathrm{kHz}$, digitized using a National Instruments A-D board, and acquired at $10 \mathrm{kHz}$ in IGOR Pro (WaveMetrics).

\section{Whole-cell patch clamp recordings}

Whole-cell patch clamp recordings were, in general, performed as described previously (Murthy et al., 2008). Modifications of the dissection were required to access the different AMMC neurons. For the A1 and GF neurons, we accessed the brain from the posterior side of the head [similar to the protocol described in the study by Murthy and Turner (2010)]. For B2 neurons, we accessed the brain through the proboscis, as these cell bodies lie ventral to the AMMC. For B1 neurons, which are positioned just above (dorsal to) the AMMC, we accessed the brain from the anterior side of the head. For all preparations, we ensured that all three segments of the antennae remained underneath the recording platform (the area above the recording platform is immersed in saline, while the area underneath is kept dry using a wax barrier to separate the two sides) (for more information on mounting flies for in vivo recordings, see Murthy and Turner, 2010). Following dissection and before recording, we checked for self-sustained vibrations of the antennae in response to gentle air flow and for spontaneous leg movements (to indicate the health of the preparation). For AMMC-A1 recordings [these neurons bilaterally innervate right and left AMMCs (Kamikouchi et al., 2009); our recordings came from neurons in either the right or left hemisphere], we used 
patch clamp electrodes pulled from capillary glass (o.d., 1.5; i.d., 1.1) with resistances of $\sim 5-6 \mathrm{M} \Omega$. The perineural sheath and overlaying neurons were typically removed on the electrophysiology rig with a cleaning pipet, and under visual control. Only neurons with input resistances $>100 \mathrm{M} \Omega$ were included for analysis. All cells were held between -55 and $-65 \mathrm{mV}$, in current-clamp mode, using either an Axoclamp 2B or a MultiClamp $700 \mathrm{~B}$ amplifier, and voltage signals were acquired in IGOR Pro at $10 \mathrm{kHz}$ via a National Instruments A-D board. Recordings typically lasted for $30-60 \mathrm{~min}$, and permitted the measurement of responses to (on average) $80 \%$ of our stimulus set.

\section{Song recordings}

We recorded $D$. mel and $D$. sim songs in a 1-cm-diameter chamber with a nylon mesh floor that rested $2 \mathrm{~mm}$ above the surface of a pressuresensitive microphone (Brüel \& Kjær 41791 inch free-field microphone); the sides of the chamber were coated with fluon, which limited the movement of flies to the floor of the chamber. Songs were recorded in a semi-anechoic box from Acoustic Systems (ETS-Lindgren), digitized via a National Instruments A-D board, and acquired at $15 \mathrm{kHz}$ in LabVIEW.

\section{Data analysis and statistics}

Analysis was performed in MATLAB (MathWorks).

\section{Calculation of response amplitudes and normalization}

For steady-state responses. (1) JO: Response amplitudes were calculated as the sum of the absolute value of the voltage signal (following baseline subtraction) in a $20 \mathrm{~ms}$ time window and averaged across all windows for the length of the steady-state response period (0.5-3.5 s after stimulus onset). (2) AMMC: Response amplitudes were calculated as the change in voltage from baseline, during the steady-state region of the response (DC component).

For onset responses (see Fig. 4F only). (1) JO: Response amplitudes were calculated as the sum of the absolute value of the voltage signal (following baseline subtraction), in a $10 \mathrm{~ms}$ time window that followed stimulus onset by $2.5 \mathrm{~ms}$. (2) AMMC: Response amplitudes were calculated as the sum of the absolute value of the voltage signal (following baseline subtraction), in an $80 \mathrm{~ms}$ time window that followed stimulus onset by $2.5 \mathrm{~ms}$.

For transient responses to pulse stimuli. (1) JO: Response amplitudes were calculated as the sum of the absolute value of the voltage signal in a $20 \mathrm{~ms}$ time window (following baseline subtraction) that followed the pulse stimulus by $2.5 \mathrm{~ms}$ (computed lag between stimulus and response). (2) AMMC: Response amplitudes were calculated as max-min in the 20 $\mathrm{ms}$ time window following the pulse stimulus by $2.5 \mathrm{~ms}$. Both JO and AMMC-A1 responses were normalized as follows: (1) for pure-tone stimuli (see Fig. 3), we found the maximum response amplitude across all frequencies $(100-900 \mathrm{~Hz})$, for a given animal and stimulus intensity (red or blue), and divided all response amplitudes (for that animal and that intensity) by this maximum; (2) for white-noise stimuli (see Fig. 4), we found the maximum response amplitude across all stimulus intensities (green, blue, and red), for a given animal, and divided all response amplitudes (for that animal) by this maximum; (3) for fly song stimuli (see Figs. 5, 6), we found the maximum response amplitude across all 612 pulse stimuli tested (from 10 song clips), for a given animal, and divided all response amplitudes (for that animal) by this maximum. After normalization, response amplitudes were averaged across animals to form frequency and intensity tuning or response curves.

\section{Calculation of response sensitivities}

For each song pulse, response sensitivity was computed as the normalized response amplitude $\left(V / V_{\max }\right)$ divided by the stimulus intensity.

\section{Calculation of FFT amplitudes}

For steady-state responses (to pure tones and white noise), FFTs were computed using sliding $20 \mathrm{~ms}$ response windows (Hamming), and then absolute values of the resulting coefficients were averaged. For pulse stimuli, FFTs were computed on the $20 \mathrm{~ms}$ window that included each pulse stimulus; for pulse responses, FFTs were computed on the $20 \mathrm{~ms}$ response window that followed the stimulus by $2.5 \mathrm{~ms}$. Normalization was performed as described above.

\section{Cross-correlations and error energies}

All correlation coefficients reported in this study come from taking the maximum computed coefficient from sliding cross-correlations (with mean subtraction of signals and with typical lags of $100 \mathrm{~ms}$; $x \operatorname{cov}$ function in MATLAB). To compute the error energy (EE) between signals $x$ and $y$, we first scaled both $x$ and $y$ between 0 and 1 , and then used the following formula: $\mathrm{EE}=\operatorname{mean}\left(\operatorname{sum}(x-y)^{2} / \operatorname{mean}\left(\operatorname{sum}(x-\operatorname{mean}(x))^{2}\right)\right.$.

Measurement of decay times and simulation of AMMC responses Pulse responses (lasting $>22 \mathrm{~ms}$ ) were extracted from recordings and only responses with response heights $>3 \times$ background noise were selected. We fit the decay of each pulse response (the $20 \mathrm{~ms}$ period that begins $2 \mathrm{~ms}$ after the peak) to a single exponential function. The fits were of the following form: $a\left(e^{-b t}\right)+c$, where $c$ is a constant term to account for the DC offset in the response. Decay time then equals $1000 / b$ (in milliseconds). To simulate AMMC responses, male IPI distributions were approximated from the study by Cowling and Burnet (1981) using kernel density estimation. For each iteration of the model, 19 IPIs were drawn from one of these distributions (D. mel, D. sim, or the hybrid) and used to construct an artificial train of 20 AMMC pulse responses. Both rise times [taken as the mean rise time of actual pulse responses (see Fig. $5 G)]$ and maximum response amplitudes $\left(V_{\max }\right)$ were fixed. That is, the maximum absolute amplitude of any pulse response was set equal to the amplitude of the first pulse response in the train, and rise times were scaled such that the maximum response amplitude was not exceeded [in agreement with our recordings (see Fig. 5G)]. Response decays were modeled as single exponentials, and no noise was added to the simulated response. For each decay time tested (from 2 to $24 \mathrm{~ms}$ ), we ran 100 iterations of the model to obtain the mean and SD of the DC offset.

\section{Statistics}

All $p$ values in this study come from either two-tailed Student's $t$ tests, assuming unequal variances or a balanced one-way ANOVA (only Fig. 4).

The adjusted $r^{2}$ statistic is a measure of the correlation between the data values and the predicted data values (based on the fit); perfect correlation $=1$. It was calculated as the ratio of the sum of squares of the regression and the total sum of squares, and adjusted for residual degrees of freedom (sample size minus the number of fitted coefficients).

\section{Results \\ Recordings from sound-sensitive interneurons of the Drosophila AMMC}

Tuning curves for JO neurons have been previously characterized using electrostatic actuation to vibrate the arista (Albert et al., 2007). This method is not, at present, compatible with patch clamp recordings; we therefore used an alternative (a small earphone speaker coupled to a length of tubing) for delivering both calibrated and localized sounds to flies during electrophysiological recordings (see Materials and Methods) (Fig. 1). Our sound delivery system was designed to mimic the singing of the male during natural courtship (Bennet-Clark, 1971), and all stimuli were concentrated in the frequency and intensity ranges of recorded $D$. mel and $D$. sim songs [for both species, roughly $100-$ $900 \mathrm{~Hz}$ and $0.1-10 \mathrm{~mm} / \mathrm{s}$ (Figs. $1 A, 5 A$ ), which is within the dynamic range of the D. mel arista (Göpfert et al., 2006)].

The auditory pathway of Drosophila begins in the antenna, where $\sim 500$ JONs send projections into the brain, to the AMMC (Kamikouchi et al., 2006). The AMMC is divided into five zones, and each JON typically innervates only one. A previous study identified four types of central neurons (based on morphology) that innervate AMMC zones A and B; these neurons were proposed to be postsynaptic to zone A and B JONs, and therefore likely responsive to courtship song (Kamikouchi et al., 2009). We consequently focused our analysis on these neurons (Fig. 2A,B); they include two neuron types that innervate zone A, GF ( 1 cell/ hemisphere) and AMMC-A1 (2 cells/hemisphere), and two neuron types that innervate zone $\mathrm{B}, \mathrm{AMMC}-\mathrm{B} 1[10.5 \pm 0.8$ cells/ 
A
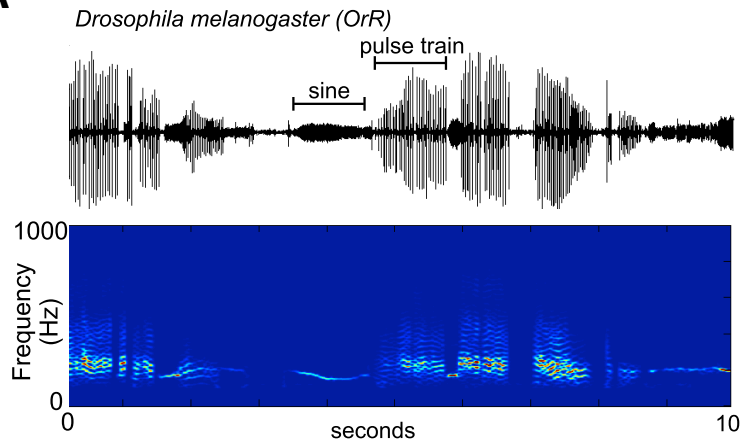

Drosophila simulans (194)
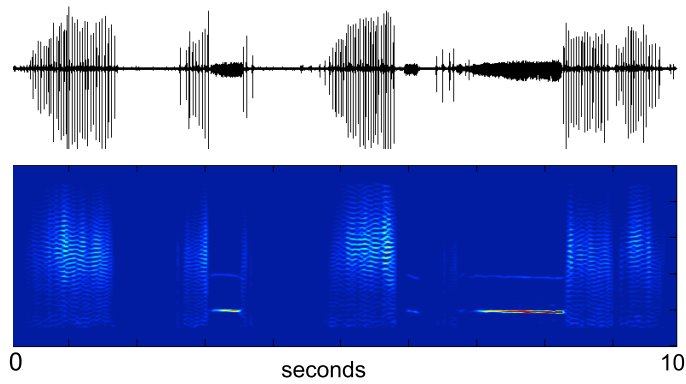

B

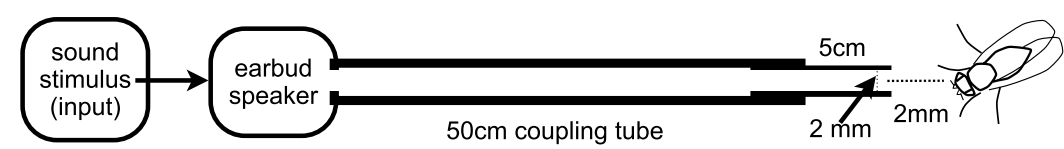

C

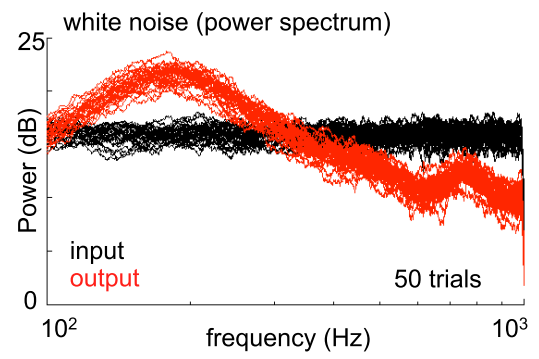

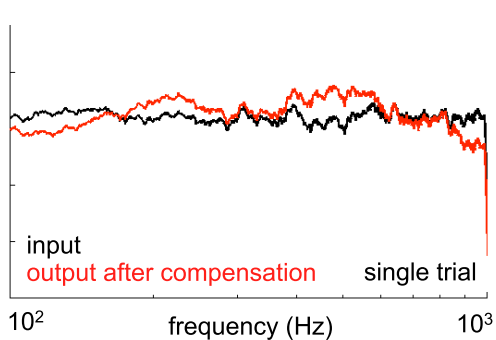

D

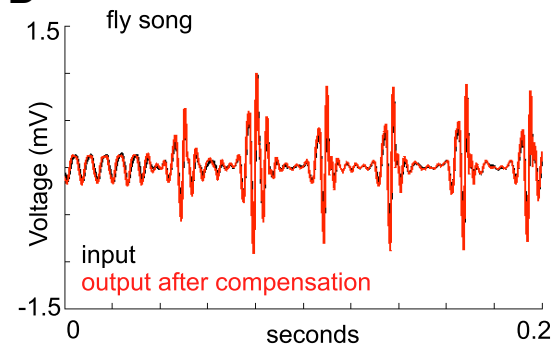

Figure 1. Courtship songs and sound delivery. $A$, Courtship song traces (each $10 \mathrm{~s}$ ) from D. melanogaster (left) and D. simulans (right). Regions of sine and pulse song are indicated. Spectrograms ( 150 ms sliding FFT window) of each trace are plotted below; song power in both D. melanogaster and D. simulans is concentrated in the $100-900 \mathrm{~Hz}$ frequency band. $\boldsymbol{B}$, Diagram of the sound delivery system. Sound (filtered in MATLAB) is played through an earbud speaker coupled to a length of polyethylene tubing. The end of the tube is placed $\sim 2 \mathrm{~mm}$ from the fly's antenna [corresponding to the approximate distance between a courting male and receptive female (Bennet-Clark, 1971)]. C, Left, Fifty trials of white noise (bandpass filtered between 80 and 1000 Hz; black curves) delivered through the sound tube without filtering produces output curves in red (recorded on a calibrated B + K microphone placed $2 \mathrm{~mm}$ from the end of the sound delivery tube). Right, Single trial of white noise (black) and the output after convolving the signal with the inverse transfer function filter (red) (see Materials and Methods). D, Recorded output (red) of convolved fly song is a near perfect match with the original song (black).

hemisphere ( $n=7$ brains)] and AMMC-B2 (2 cells/hemisphere). Antibody staining suggests that AMMC-B1 neurons are cholinergic and AMMC-B2 neurons are GABAergic (Fig. $2 B$, inset); AMMC-A1 and GF neurons may use other neurotransmitters, because neither neuron type was found to be choline acetyltransferase (ChAT) or GABA immunoreactive (data not shown). Zone A AMMC neurons are part of the giant fiber pathway, which senses both visual and mechanosensory stimuli to trigger an escape response; these two types of neurons are also gap junction coupled to one another (Phelan et al., 2008) (and our dye fills). While the specific inputs to the giant fiber pathway are not known, in wild-type flies, the GF neuron is not activated by either visual or mechanosensory stimuli alone (Engel and $\mathrm{Wu}, 1996$; Fotowat et al., 2009).

Using well established methods for in vivo whole-cell patch clamp in flies (Wilson et al., 2004; Murthy and Turner, 2010), we recorded from at least two animals for each of these AMMC neuron types and found in all cases graded responses to sound pulses; these potentials are tightly locked to the sound stimulus, and we observed no differences in temporal delay between sound stimulus and graded response across the different AMMC neurons (Fig. 2C). Depolarizing current injection (steps from -60 to $0 \mathrm{mV}$ ) at the soma of AMMC-A1 or -B2 neurons (during current clamp recordings) failed to elicit action potentials in these cells (data not shown). Because neuronal morphologies of these cells are different (Fig. $2 B$ ), it is unlikely that our inability to control the membrane potential at sites distant from the soma affected our ability to generate and observe spikes via current injection. In contrast, current injection at the GF neuron soma produced a large spike [Fig. 2 D; resembling a typical $\mathrm{Ca}^{2+}$ spike, found commonly in insect "nonspiking" neurons (Laurent, 1991)], again indicating that the small-amplitude graded responses to sound, observed uniformly across these four cell types, are likely not an artifact of membrane filtering of spiking events occurring distal to the soma. To test this further, we asked whether or not sound responses could summate with subthreshold somatic current injection to drive spiking in the GF neuron. Playing sound simultaneously with current injection that, on its own, was not able to elicit a spike in the GF neuron, produced a full-blown spike (Fig. $2 D)$. As sound responses were qualitatively similar across these four types of neurons (Fig. 2C), we focused the remainder of our analyses on the AMMC-A1 neurons, which proved more accessible for patch clamp recordings.

We then measured response reliability of JO and AMMC-A1 neurons by analyzing responses to 10 different clips of courtship song (Fig. $2 E$ ). JON population responses were similar whether recorded through the antenna or with a suction electrode to access the antennal nerve directly as it enters the brain (data not shown); that is, local field potential (LFP) recordings did not simply reflect antennal muscle activity. AMMC-A1 neuronal responses are highly correlated across animals: correlation coefficients for comparisons across animals and for each song stimulus 


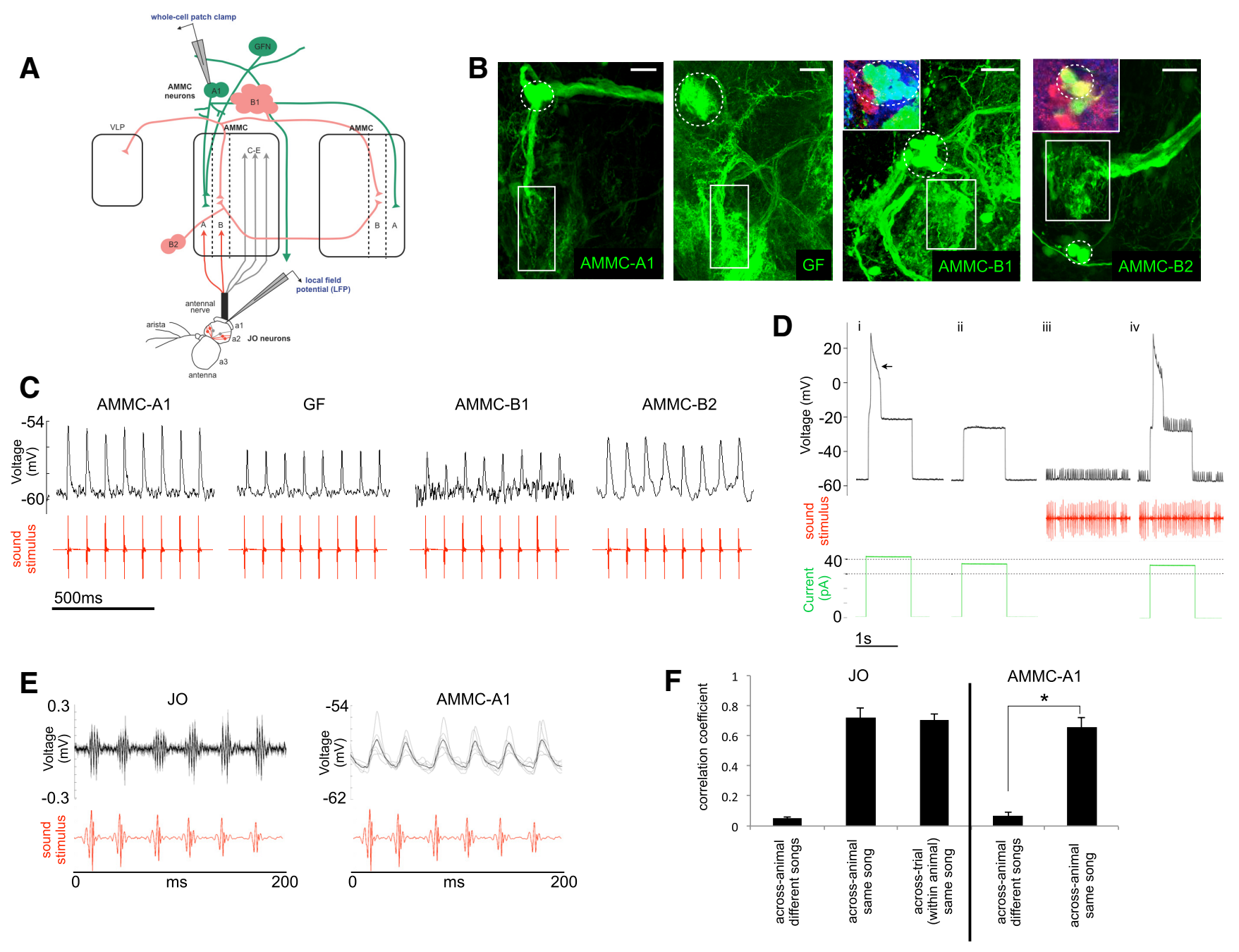

Figure 2. Recordings from AMMC neurons. A, Diagram of the early auditory system: $\sim 500 \mathrm{JONs}$ from the second segment of the antenna (a2) project into the brain (50 - $100 \mathrm{JONs}$ to zone A and $100-150$ JONs to zone B). We used four different GAL4 lines (see Materials and Methods) to label four classes of central neurons that innervate the AMMC (for more information on these classes, see Kamikouchi et al., 2009). The number of neurons per hemisphere labeled in each line are indicated [AMMC-A1 (2 cells), GF (1 cell), AMMC-B1 (10 cells), and AMMC-B2 (2 cells)], as well as the location of electrode placements to record from the JONs (extracellular) and AMMC neurons (intracellular). B. Confocal images ( $z$ projections) of the four GAL4 lines recorded from in this study. Cell bodies are indicated with a dashed circle, and the area of neurite innervation in the AMMC neuropil (only a portion of one hemisphere is shown) with a solid white box. Scale bars, $20 \mu \mathrm{m}$. Insets, Colocalization of antibodies to GABA (red) or ChAT (blue) with GFP (green) for two of the GAL4 lines (cell bodies of AMMC neurons circled). C, Representative intracellular recordings from each of the four types of AMMC neurons ( $n \geq 2$ for each); responses (black) to D. mel sound pulses spaced (artificially) with 90 ms interpulse intervals (red). D, Representative responses (black) recorded from the GF neuron soma to (i) suprathreshold current injection (which elicits a spike, indicated with an arrow), (ii) subthreshold current injection, (iii) D. mel courtship song, and (iv) subthreshold current injection plus D. mel courtship song (this combination also elicits a spike). $\boldsymbol{E}$, Reliability of sound responses across animals: pulse song responses in AMMC-A1 neurons (right) from six flies (gray), overlaid with the average (black). For comparison, JON recordings from six $D$. mel flies is shown on the left (gray), overlaid with the average (black). $\boldsymbol{F}$, Mean-subtracted cross-correlation of responses to 10 different fly song clips [across animals and trials; $n=6 \mathrm{JON}$ recordings (with 2-8 trials/animal) and $n=8 \mathrm{AMMC}-\mathrm{A} 1$ recordings]. Song responses are as correlated across animals as they are across trials within an animal $\left({ }^{*} p=4.3 \times 10^{-10}\right.$, from a Student's $t$ test). Error bars are SEM.

are significantly larger than correlation coefficients for comparisons between different song clips (Fig. $2 F$ ). As we were unable to record multiple trials for every song stimulus during AMMC-A1 patch clamp recordings (due to the size of our auditory stimulus set and often short duration of recordings), we compared acrossanimal correlation coefficients with those from JON LFP recordings, and found a similar degree of correlation in fly song responses across animals and across trials within an animal (Fig. $2 F$ ). This reproducibility in neural responses (both at the level of the JON population and the AMMC-A1 neurons) enabled us to form tuning curves across experiments for the three different types of stimuli we examined: pure tones, white noise, and fly song.
Steady-state responses: differences in frequency and intensity tuning between the Johnston's organ in the antenna and AMMC-A1 neurons in the brain

To examine the frequency tuning of the JON population and AMMC-A1 neurons, we recorded responses to tonal stimuli in the frequency range of $D$. mel and D. sim courtship songs (100$900 \mathrm{~Hz}$ ) and at two particle velocity levels (Fig. 3A). Because a given animal may have received tonal stimuli of only one of the two stimulus intensities (red or blue), we normalized tuning curves $\left(V / V_{\max }\right)$ for each stimulus intensity and each animal separately (thus, first we focused on only frequency tuning). JON LFP response amplitudes during the steady-state period (0.5-3.5 $\mathrm{s}$ following stimulus onset; Fig. $3 B$ ) were maximal for tones of 

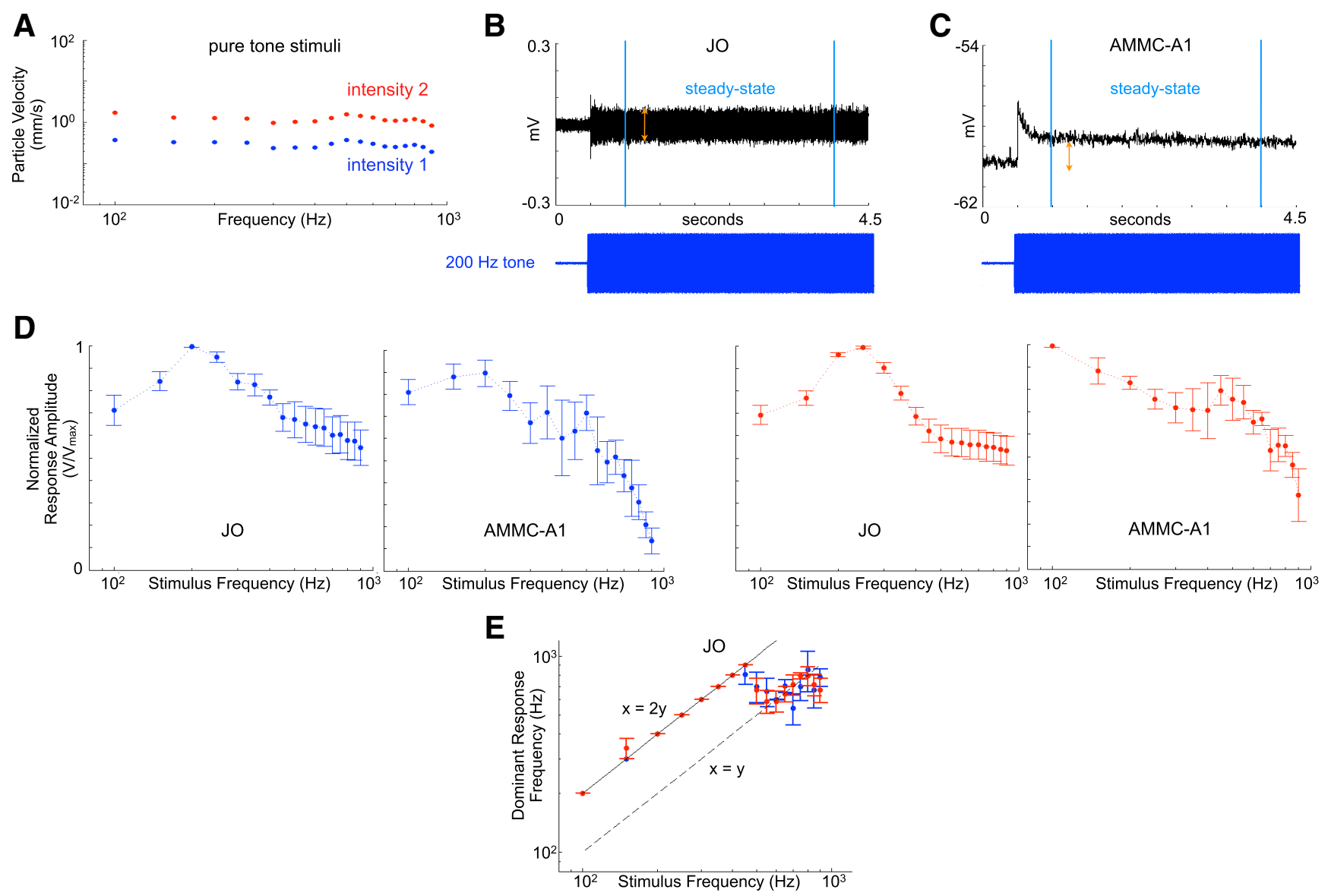

Figure 3. Responses to pure tones. $\boldsymbol{A}$, Frequency and intensity spectra of sine wave stimuli. $\boldsymbol{B}$, Representative JON response to a $200 \mathrm{~Hz}$ tone delivered at intensity 1 . The steady-state period of the response is indicated; this period was used for all subsequent analyses. Response amplitudes were calculated as average peak-peak voltages (orange arrow), after baseline subtraction and during the steady-state period. C, Representative AMMC-A1 neuron response (whole-cell patch clamp recording) to the same stimulus as in $\boldsymbol{B}$. Response amplitudes were calculated as the average change in membrane voltage from baseline (orange arrow) during the steady-state period. $\boldsymbol{D}$, Normalized $\left(V / V_{\max }\right)$ JON and AMMC-A1 neuron response amplitudes; normalization was done separately for the two stimulus intensities (error bars are SEM) (JON recordings: $n=9$ flies; AMMC-A1 recordings: $n=7$ flies). E, Dominant JON response frequency (the frequency at which the amplitude of the FFT of the steady-state response was greatest) plotted against the stimulus frequency. Error bars are SEM.

$\sim 200 \mathrm{~Hz}$ (Fig. 3D), consistent with tuning curves produced for the arista by laser Doppler vibrometry (Göpfert and Robert, 2002). JON responses are oscillatory; we observed that the dominant frequency of the JON response is twice the input frequency, for stimulus frequencies up to $\sim 450 \mathrm{~Hz}$ (Fig. 3E), consistent with the known mechanical structure of the JO [two populations of JONs each responding to one phase of the tonal stimulus (Nadrowski et al., 2008)]. The AMMC-A1 neurons innervate a subregion of AMMC zone A, which contains synapses from $\sim 50-100$ JO-A neurons (Kamikouchi et al., 2006). AMMC-A1 neurons produce a sustained depolarization during the pure tone stimulus (Fig. 3C); the amplitude of this depolarization decreases with increasing stimulus frequency (particularly past $600 \mathrm{~Hz}$ ), and, in contrast to the JON population, tuning curves do not show a peak for $\sim 200 \mathrm{~Hz}$ stimuli (Fig. 3D).

Broadband stimuli such as white noise can be useful to characterize the response of a system to a large range of frequencies simultaneously. We therefore used white noise (band-pass filtered between 80 and $1000 \mathrm{~Hz}$ ) to examine both frequency response and intensity tuning curves for the JO population and AMMC-A1 neurons. We stepped the intensity of white noise from roughly 0.1 to $3 \mathrm{~mm} / \mathrm{s}$ in three steps (Fig. $4 A$ ), to span a range of intensities flies typically encounter during courtship (Bennet-Clark, 1971). We first examined FFT amplitudes for
JON population and AMMC-A1 neuron responses during the steady-state response period. FFT amplitudes for the JON population reveal a peak at $\sim 300 \mathrm{~Hz}$ (Fig. 4D), largely consistent with tuning curves formed with tonal stimuli (tuning curves were peaked for $200 \mathrm{~Hz}$ stimuli, which produce a $\sim 400 \mathrm{~Hz}$ response in the JONs; Fig. 3). However, we observed that FFT amplitudes decreased with increasing stimulus intensity. This same trend could be observed when we instead calculated response amplitudes across stimulus intensities (Fig. $4 F$ ) or response variance (data not shown).

To examine the responses of the AMMC-A1 neurons, we first subtracted the DC component of the response and computed the FFT of the voltage fluctuations riding on top of the DC response to white noise. In contrast to the JON population, FFT amplitudes for AMMC-A1 neurons are identical across stimulus intensities (Fig. 4E). That is, while DC-subtracted activity is boosted above baseline during the steady-state response period, it does not vary across stimulus intensities. We also examined the DC component of the AMMC-A1 response to white noise and found, in contrast to the JON population, no significant trend with increasing stimulus intensity (Fig. $4 F$ ). These data collectively reveal a transformation in both frequency and intensity tuning between the JON population and AMMC-A1 neurons during the steady-state response period. Given that a previous study dem- 
A

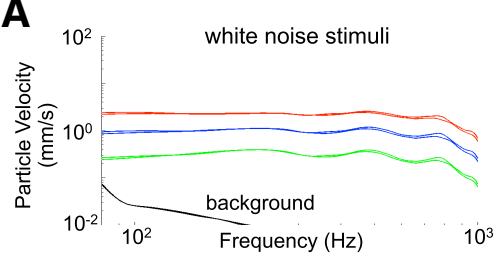

B
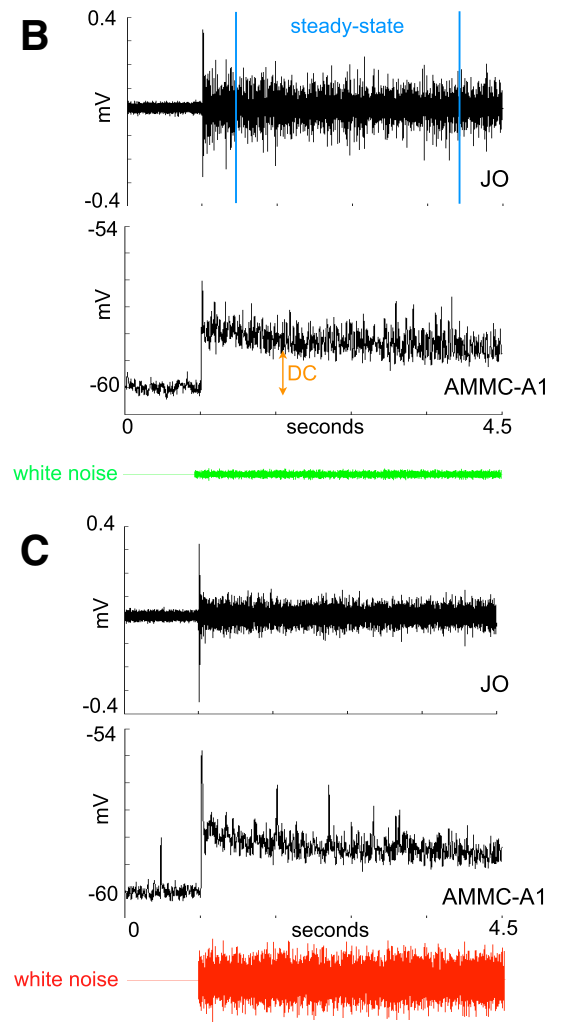

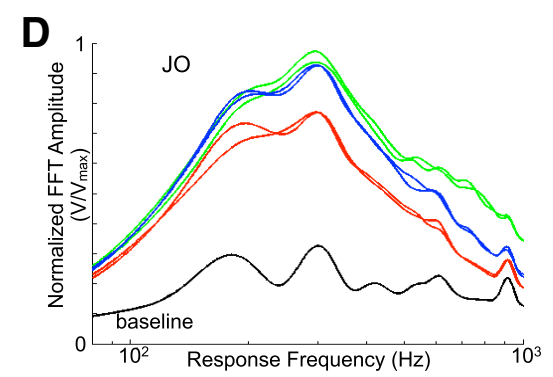

E
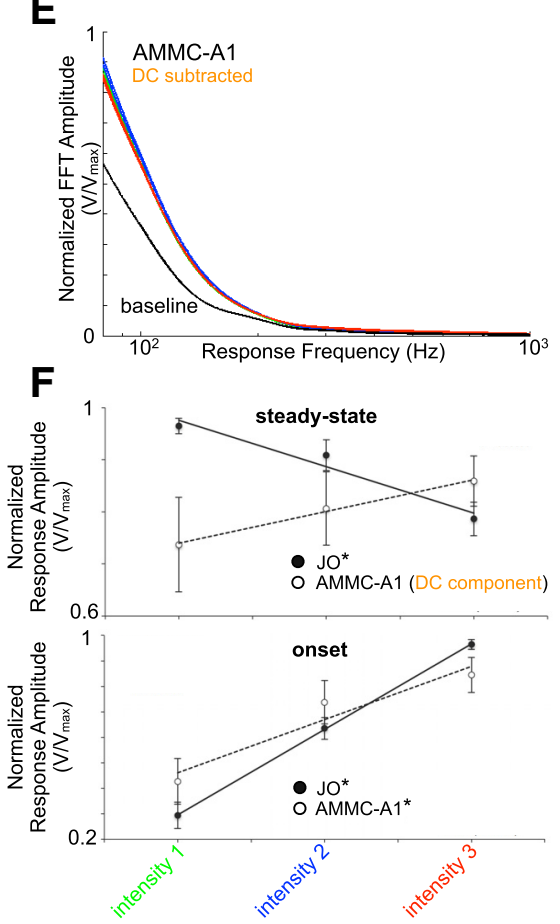

Figure 4. Responses to white noise. $A$, Frequency and intensity spectra of the six white-noise (WN) stimuli (2 variants of each intensity: green, blue, red); intensity was increased in steps of $\sim 10 \mathrm{~dB}$. B, Representative JON (top) and AMMC-A1 neuron (bottom) responses to the lowest intensity WN stimulus (green). The steady-state period of the response is indicated; this period was used for all subsequent analyses (except onset responses in $\boldsymbol{F}$ ). The DC component of the AMMC-A1 neuron response (indicated) was subtracted for the analysis presented in $\boldsymbol{E}$. $\boldsymbol{C}$, Representative JON and AMMC-A1 neuron responses to the highest intensity WN stimulus (red). $\boldsymbol{D}$, Average JON frequency response curves $(n=8)$ to the stimuli depicted in $\boldsymbol{A}$; FFT amplitudes were normalized for each experiment and then averaged across experiments. Average FFT amplitude of baseline (before stimulus onset) is shown in black. $E$, Average AMMC-A1 neuron (DC-subtracted) frequency response curves $(n=7)$ to the stimuli depicted in $A$; FFT amplitudes were calculated as in $\boldsymbol{D}$. $\boldsymbol{F}$, Top, JON and AMMC-A1 neuron normalized response amplitudes during the steady-state period, as a function of intensity of white-noise stimuli. Linear fits to the data yield slopes of -0.09 for the $\mathrm{J} 0\left(r^{2}=0.96\right)$ and 0.06 for AMMC-A1 neurons $\left(r^{2}=0.99\right)$. A balanced one-way ANOVA was used to compare differences in response amplitude across intensities (for JON, ${ }^{*} p=2 \times 10^{-6}$; for AMMC-A1, $p=0.3$ ). Bottom, In contrast to at steady state, both JON and AMMC-A1 neuron response amplitudes to stimulus onset increase linearly with increasing stimulus intensity. Linear fits yield slopes of 0.34 for the JO $\left(r^{2}=1\right)$ and 0.21 for AMMC-A1 neurons $\left(r^{2}=0.93\right)$. For a balanced one-way ANOVA: JON, ${ }^{*} p=1 \times 10^{-16}$; AMMC-A1, ${ }^{*} p=4 \times 10^{-5}$. All error bars are SEM.

onstrated that the JO population response to the frequencies and intensities we examined is dominated by the activity of the JO-A neurons, as opposed to JO-B or JO-CE neurons (Kamikouchi et al., 2009), it seems likely that the transformation we observe reflects a difference between AMMC-A1 neurons and JO neurons that also innervate zone A (see Discussion).

\section{Transient responses: AMMC-A1 neuron responses to} courtship song pulses are matched to the JON population Above, we characterized auditory tuning during the steady-state response period. We noticed, however, that responses were often maximal at stimulus onset (Figs. $3 B, C, 4 B, C$ ). Stimulus onsets have obvious ethological relevance, and nearly all auditory neurons stud- ied respond briskly to onsets (Heil, 1997a,b). All of our white-noise stimuli contained a $10 \mathrm{~ms} 3 \mathrm{kHz}$ pulse at onset (followed immediately by white noise); analysis of onset responses across the three intensities we tested revealed a surprising match between the JON population and AMMC-A1 neurons. At onset, both responses increase linearly with intensity (Fig. $4 F$ ). We reasoned that transient responses to courtship song pulses (characteristically 5-20 ms pulses spaced with species-typical interpulse intervals) might therefore also be matched between the JON population and AMMC-A1 neurons.

Because D. mel females will copulate not only with $D$. mel males, but also with D. sim males (Sturtevant, 1920), we chose to make comparisons between these two closely related species [separated by only 2-3 million years of evolution (Clark et al., 2007)]. Our song stimuli therefore consisted of 612 pulses presented within 10 clips of either $D$. mel or $D$. sim song (Fig. 5A); songs from these two species are distinct: $D$. sim songs contain higher average pulse carrier frequencies $(480 \mathrm{~Hz}$ in $D$. sim compared with $280 \mathrm{~Hz}$ in $\mathrm{D}$. $\mathrm{mel}$ ) and larger average IPIs ( $55 \mathrm{~ms}$ in D. sim compared with $30 \mathrm{~ms}$ in $\mathrm{D}$. mel) (Cowling and Burnet, 1981). We first used these stimuli to compare pulse song responses between the JON population and AMMC-A1 neurons in D. mel.

In contrast to responses at steady state, tuning for fly song pulses was similar between the JON population and AMMC-A1 neurons. With increasing pulse intensity, we observed an increase in response amplitude and a decrease in sensitivity (Fig. 5B). Fitting the data with a straight line [goodness of fit (adjusted $r^{2}$ ) for $\mathrm{JONs}=0.9$; and for AMMC-A1 neurons $=0.8]$ yielded nearly identical slopes for JONs $(-0.9)$ and AMMC-A1 neurons $(-0.8)$. The relatively flat relationship between intensity and response amplitude reveals that the softest pulses in our stimulus set evoked almost as robust a response as the loudest pulses (Fig. 5D). These results are consistent with JO LFP (also known as compound action potential) onset responses measured using electrostatic forcing to displace the arista and over a similar range of stimulus intensities (Albert et al., 2007). We also compared frequency tuning for pulse stimuli. JON population tuning was flat within the range from 100 to 900 $\mathrm{Hz}$ (in contrast to at steady state), and, again, was matched to AMMC-A1 neuron tuning [Fig. 5C; response sensitivities are not dependent on frequency, and therefore form the mirror image (inverse) of the frequency and intensity profiles of the stimuli themselves, plotted in Fig. 5A].

The similarity between JON population and AMMC-A1 neuron responses to fly song pulses is perhaps unexpected, given the 
A

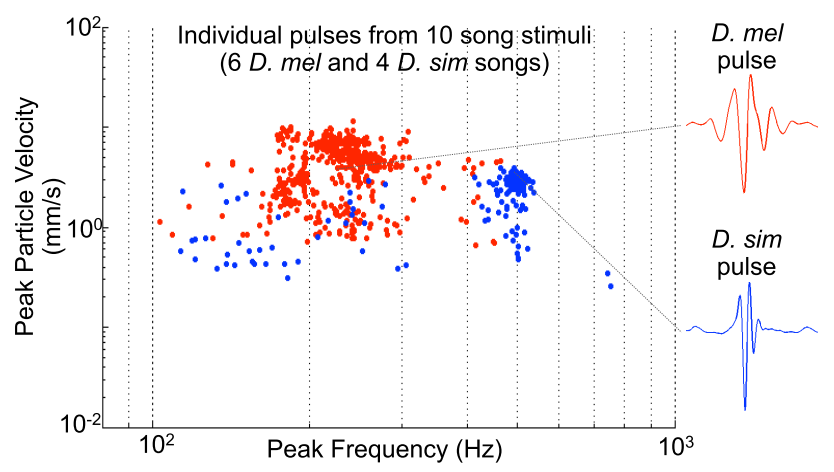

D
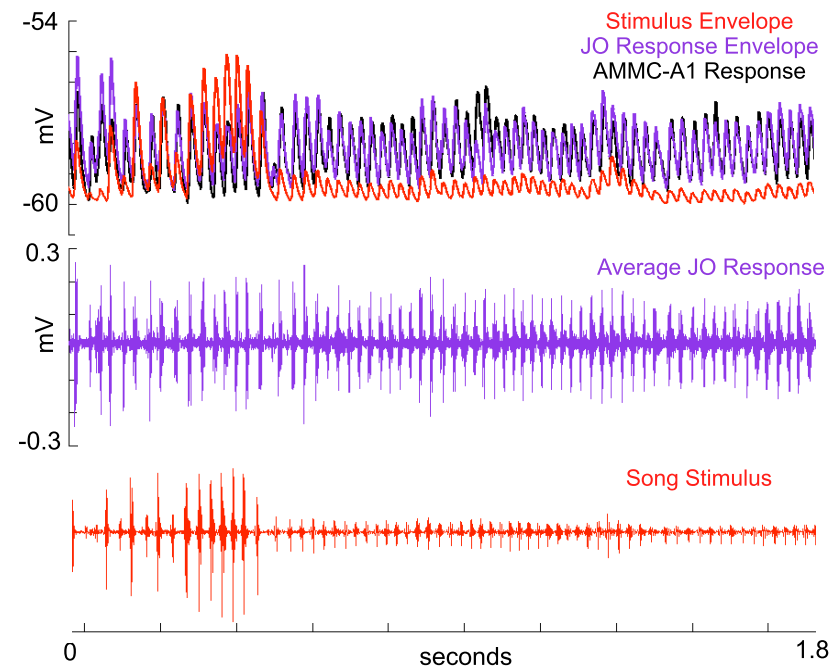

F

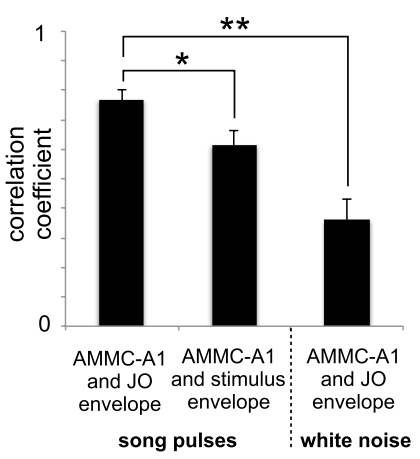

B
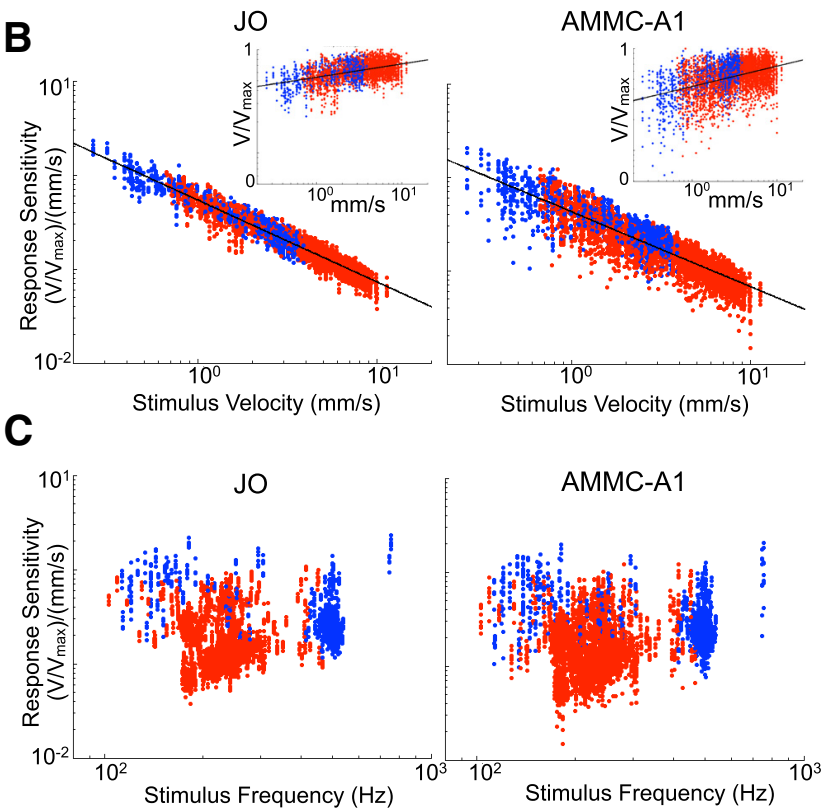

E

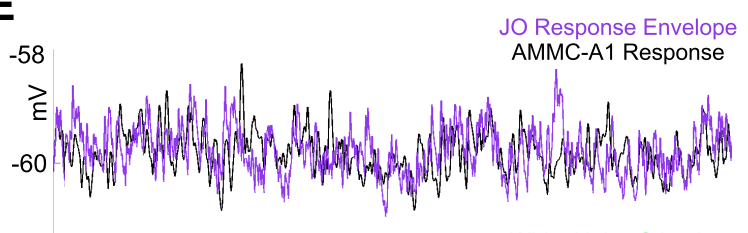

White Noise Stimulus

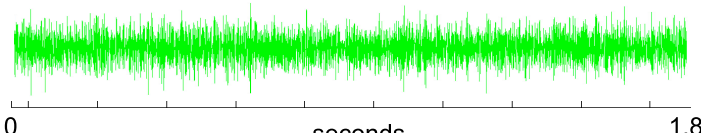

G
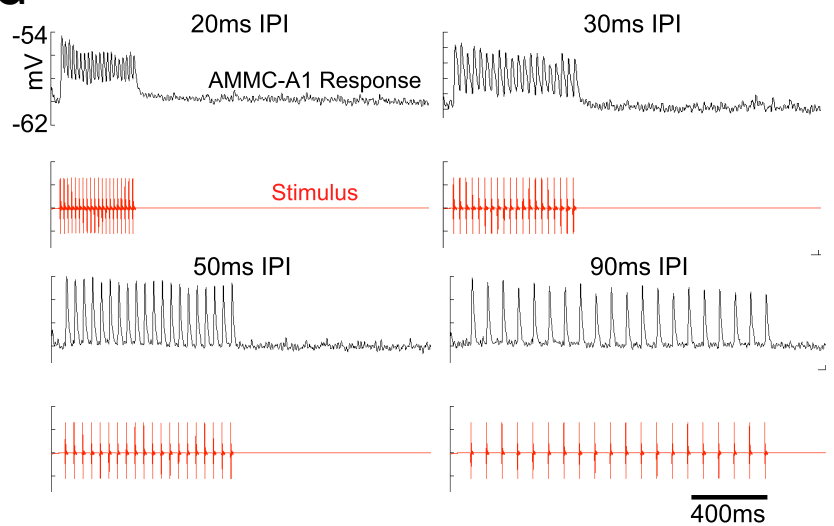

Figure 5. Responses to courtship song pulses. $A$, Peak frequency and intensity for 612 individual pulses (dots) extracted from the 10 song stimuli ( 6 songs from $D$. mel and 4 songs from $D$. sim). In our recordings, D. mel pulses (red) are typically lower in frequency, but higher in intensity than those from $D$. sim (blue). Examples of individual pulses shown on the right are 30 ms in length. $\boldsymbol{B}$, Pulse response sensitivities decrease as a function of stimulus particle velocity for both the J0Ns (left; $n=6$ ) and AMMC-A1 neurons (right; $n=8$ ); response sensitivity is the normalized response amplitude $\left(V / V_{\max }\right)$ divided by the stimulus particle velocity (in millimeters per second) for the $20 \mathrm{~ms}$ response period following each pulse stimulus shown in $\boldsymbol{A}$. Plotted here are responses from the entire data set: blue dots represent responses to D. sim pulses, and red dots represent responses to D. me/ pulses. These data were fit with straight lines (black; 0 : slope $=-0.87$ and adjusted $r^{2}=$ 0.92; AMMC-A1: slope $=-0.80$ and adjusted $r^{2}=0.79$ ). Insets, $V / V_{\max }$ (normalized response amplitude) increases linearly with stimulus intensity. $\boldsymbol{C}$, Pulse response sensitivities (as in $\boldsymbol{B}$ ), as a function of stimulus frequency. $D$, Comparison of an AMMC-A1 neuron response (black) to filtered (time constant, 10 ms) versions of the $D$. me/ song stimulus (red) or average J0N response (purple). The envelopes of the stimulus (red) or average JON response (purple) were normalized such that the maximum amplitude during the $4 \mathrm{~s}$ period following stimulus onset was equal to the maximum AMMC-A1 response amplitude during that same period. $\boldsymbol{E}$, Comparison of an AMMC-A1 neuron steady-state response (black) to the filtered (time constant, 10 ms) version of the average J0N response (purple) to the lowest intensity white-noise stimulus (green, below). Both traces were mean subtracted (DC component removed). $\boldsymbol{F}$, Left, Song pulses: correlation coefficients between AMMC-A1 responses ( $n=8$ animals and 10 song stimuli per animal) and J0 response envelopes or stimulus envelopes; white noise: correlation coefficients between AMMC-A1 responses ( $n=7$ animals and 6 white noise stimuli per animal) and 0 response envelopes. All signals were first mean subtracted before performing correlations. Error bars are SEM. ${ }^{*} p=0.03$ and ${ }^{* *} p=0.0007$, from a Student's $t$ test. Right, Error energies (see Materials and Methods) between AMMC-A1 responses and the J0 response envelope for that stimulus (left) compared with the envelope of the stimulus (right). Difference in error energies between the two groups is significant ( $p=0.009$ ). G, Representative responses from an AMMC-A1 neuron to synthetic pulse trains (red), consisting of IPIs from 20 to $90 \mathrm{~ms}$ 
A

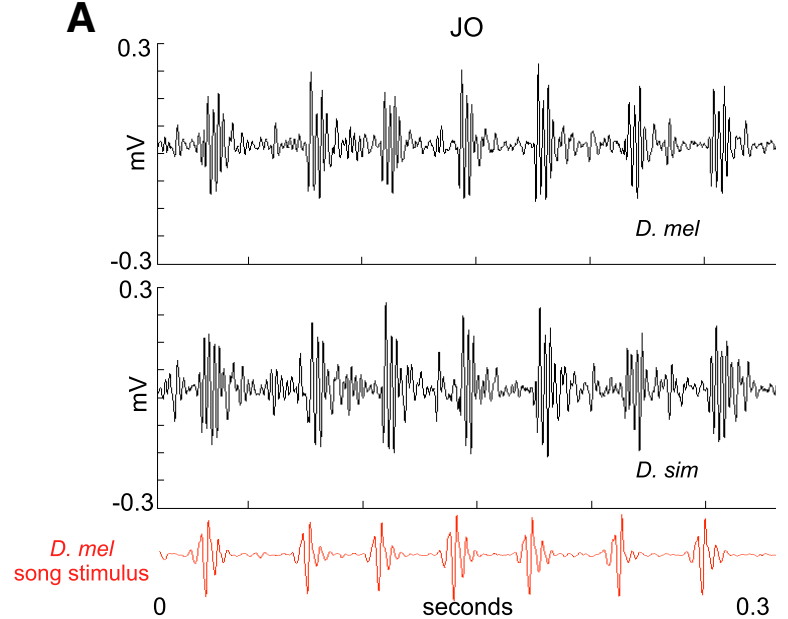

D
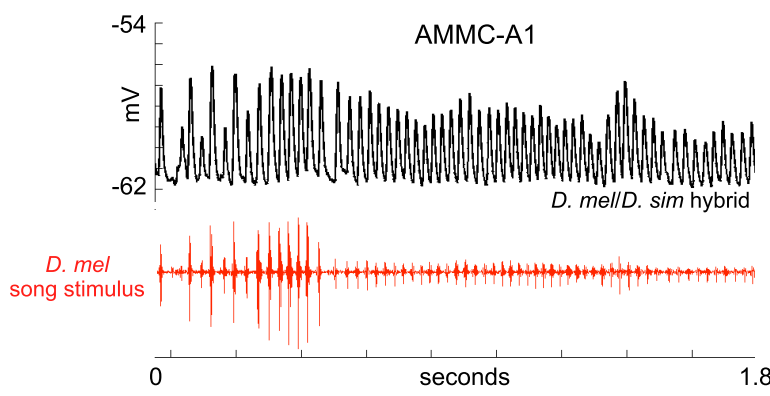

B

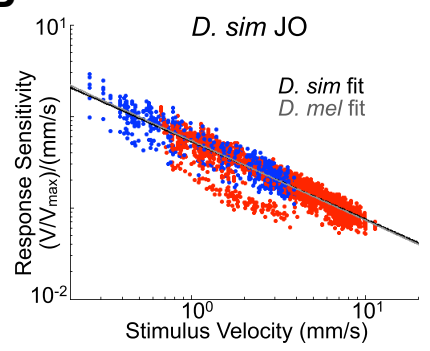

E
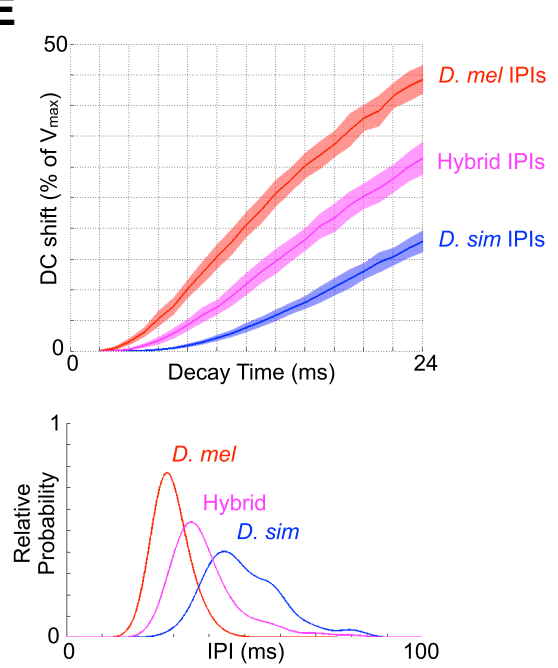

C
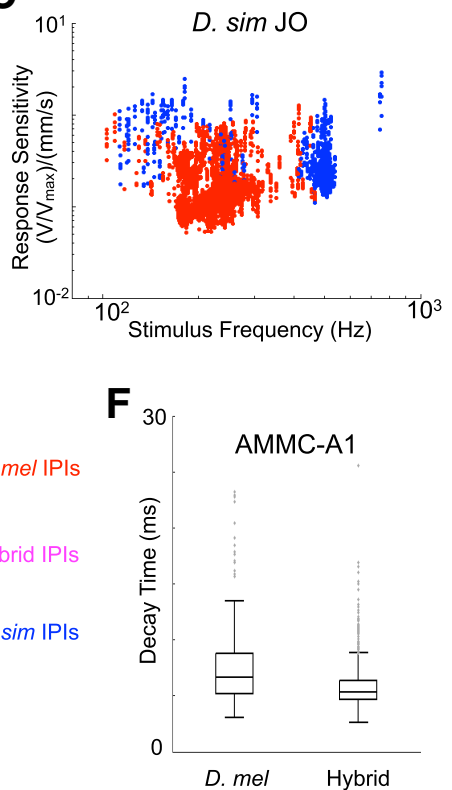

Figure 6. Comparison between D. mel and D. sim pulse responses. $A$, Representative D. mel (top) and D. sim (bottom) JoN population responses to a D. mel song stimulus (red). $B$, Similar to D. mel, pulse response sensitivities in $D . \operatorname{sim}(n=6)$ also decrease as a function of stimulus particle velocity. Plotted here are responses from the entire data set: blue dots represent responses to $D$. sim pulses and red dots represent responses to $D$. mel pulses. These data were fit with a straight line (black; slope $=-0.85$ and adjusted $r^{2}=0.87$ ). For comparison, the fit of $D$. mel pulse response data from Figure $5 B$ is overlaid in gray. $C, D$. sim pulse response sensitivities as a function of stimulus frequency. $D$, Whole-cell patch-clamp recording of an AMMC-A1 neuron in a $D$. mel/D. sim hybrid female - compare to Figure 5D. E, Top, Simulation of AMMC responses to pulse trains containing IPIs drawn from the distribution present in D. mel, D. sim, or hybrid songs (see Materials and Methods) reveals the relationship between decay times (time to reach $37 \%$ of the decay) and DC shifts of the membrane potential (plotted here as a percentage of the maximum amplitude reached, $V_{\text {max }}$ ). Means and SEM are shown with solid lines and lighter shading, respectively. Given the longer IPIs in hybrid and $D$. sim songs, correspondingly longer decay times would be required in the hybrid and D. sim neurons to achieve comparable AMMCDC shifts to those in D. mel. Bottom, IPI distributions reproduced from the study by Cowling and Burnet (1981). F, Decay times from 433 pulses responses in D. mel (left; $n=4$ ) or 1428 pulse responses in the hybrid (right; $n=2$ ) AMMC-A1 neurons [we found that decay times are independent of pulse frequency (data not shown); included here are responses to pulses from both $D$. mel and $D$. sim song]. The distance between the top and bottom of each box represents the interquartile range, and the horizontal line represents the median. Outliers (gray dots) are $>1.5 \times$ the interquartile range.

differences observed in steady-state responses to synthetic stimuli across a similar intensity range. The nature of this similarity suggested that the graded response of the AMMC-A1 neurons may be the result of a low-pass filter applied to the JON population response to fly song. We found that a first-order filter with a time constant $(\tau)$ of $10 \mathrm{~ms}$ applied to the averaged JON fly song responses (termed JO response envelopes) matched the AMMC-A1 neuron data extremely well (Fig. 5D). To quantify the match, we computed cross-correlations between each AMMC-A1 neuron response to each of the 10 song stimuli and the corresponding JO response envelope. Correlation coefficients were high (mean, 0.77) between AMMC-A1 responses and JO response envelopes, and significantly larger than between AMMC-A1 responses and stimulus envelopes (Fig. $5 F$ ). That is, the intermediate filter of the JON population response is required to model the AMMC-A1 neuron response. In further support of this, the error energy, a measure of the difference between two signals after scaling (see Materials and Methods), is significantly lower for comparisons between AMMC-A1 responses and JO response envelopes than for comparisons between AMMC-A1 responses and stimulus envelopes (Fig. $5 F$ ). The time constant of the low-pass filter (with cutoff at $\sim 50 \mathrm{~Hz}$ ) is within the biologically plausible range of membrane time constants, and suggests that filtering is, for the most part, passive. Similar to responses to fly song (Fig. 2 F), JON white-noise responses are reliable across animals (data not shown); we therefore took the average JON response to white noise and passed it through the same low-pass filter to compare AMMC-A1 white-noise responses with JO response envelopes; we found that the same filter applied to JON white-noise responses at steady state was a less reasonable fit to the AMMC-A1 data (Fig. $5 E, F)$. Thus, for transient responses specifically, AMMC-A1 responses are well matched to the population-level activity of the JO.

The graded responses of the AMMC-A1 neurons enable summation between song pulses that arrive close together, producing a DC shift (sustained membrane depolarization) in the AMMC-A1 response. To determine whether such a shift was correlated with the $D$. mel IPI range (Fig. $6 E$ ), we measured AMMC-A1 neuron responses $(n=3)$ to synthetic pulses spaced 20-90 ms apart. Graded response peaks to individual pulses are distinguishable down to an IPI of $20 \mathrm{~ms}$ (the AMMC-A1 neurons therefore preserve pulse timings in their responses), but a DC shift in the response occurs consistently below an IPI of $50 \mathrm{~ms}$ (Fig. 5G). If this DC shift is sufficient to support neurotransmitter 
release, it may serve as one mechanism for signaling the preferred IPI (see Discussion).

\section{Similar pulse song responses in D. melanogaster and D. simulans}

We next explored whether the early auditory systems of $D$. mel and D. sim could be used to differentiate between their speciesspecific pulse songs. While broadband stimuli produced a slight rightward shift (to higher frequencies) in steady-state response curves in $D$. sim females [data not shown; but consistent with the study by Riabinina et al. (2011)], tuning for pulses was indistinguishable between the two species (Fig. 6A-C). That is, normalized JO pulse response amplitudes, relative to either the intensities (Fig. $6 \mathrm{~B}$ ) or frequencies (Fig. $6 \mathrm{C}$ ) of pulse song stimuli, are nearly identical in D. mel and D. sim. This implies that these sibling species, at the level of the auditory receptor population, are equally sensitive to both $D$. mel and $D$. sim pulse songs. What happens at the next layer? While identification of AMMC-A1 neurons in D. sim is not feasible without appropriate genetic tools in that species, matings between $D$. mel females and D. sim males produce hybrid female progeny that carry the GAL4/UAS transgenes (for neural labeling) from their D. mel parent. We recorded from AMMC-A1 neurons in D. mel/D. sim hybrid females, and observed similar song responses to those from $D$. mel flies (Fig. 6D).

We observed a consistent DC shift in AMMC-A1 responses to pulse trains containing short IPIs ( 20 or 30 ms; Fig. $5 G$ ); we wanted to next explore how neuronal decay times and stimulus IPIs interacted to generate DC shifts. To do this, we generated a model and then compared this model to measured decay times from hybrid and D. mel AMMC-A1 neurons. We first established that AMMC-A 1 decays could be fit with single exponentials $\left[r^{2}=\right.$ $0.947 \pm 0.001$ (SEM)], by examining $D$. mel responses to pulses $(200-400 \mathrm{~Hz})$ separated by IPIs $>50 \mathrm{~ms}$ (these responses decayed back to baseline). We then simulated AMMC responses to pulse trains by drawing from the pulse song IPI distributions of D. mel, D. sim, or their interspecific hybrid (Cowling and Burnet, 1981) and varying single exponential decay times (Fig. 6E) (see Materials and Methods for details on the model). Pulse trains containing IPIs pulled from the D. mel distribution produced DC shifts $>5 \%$ of the maximum response amplitude with decay times (time to reach $37 \%$ of the decay) of at least $6 \mathrm{~ms}$; this $\tau$ falls squarely within the range of decay times measured from $D$. mel AMMC-A1 fly song responses (Fig. $6 F$ ). The time constant (10 $\mathrm{ms}$ ) of the low-pass filter used to convolve JO responses (Fig. 5) also falls within this range. Thus, the decay time of $D$. mel AMMC-A1 neurons is well suited for responding (with a DC shift) selectively to $D$. mel pulse song. For D. sim AMMC-A1 neurons to produce a comparable DC shift in response to $D$. sim pulse trains would require changing the decay time to $>13 \mathrm{~ms}$ (Fig. 6E); however, such a lengthening of decay time would also serve to increase the response to $D$. mel song, which may be undesired. Analysis of 1428 pulse responses from hybrid AMMC-A1 recordings did not reveal such a shift in decay times (Fig. $6 F$ ). While the D. mel phenotype may be dominant in the hybrid, our results suggest that sustained depolarization of AMMC-A1 neurons [and likely other AMMC neurons that also produce graded responses to courtship song (Fig. 2C)] occurs in both species for IPIs in the D. mel range. We discuss below how such similarity in the early auditory systems of D. mel and D. sim may be exploited for song detection in these sibling species.

\section{Discussion}

In this study, we report on the first recordings from central auditory neurons in the Drosophila brain. We find that AMMC neurons produce graded potential (nonspiking) responses to sound. While most neurons thus far characterized in the Drosophila brain (primarily in the olfactory and visual pathways, but also using whole-cell patch clamp methods) can produce action potentials in response to sensory stimulation (Wilson et al., 2004; Joesch et al., 2008), graded transmission is common in insect nervous systems, where neurite lengths are often short relative to neurite length constants (Burrows and Siegler, 1978; Büschges, 1995). Graded transmission is also used at some vertebrate central synapses (Alle and Geiger, 2006; Shu et al., 2006). For very dynamic signals, graded responses have been shown to encode more information than spiking responses (Kretzberg et al., 2001) and can be more reliable, as they do not suffer from problems associated with action potential failures. However, it remains to be determined whether and how as of yet unidentified downstream neurons in the Drosophila auditory circuit read out graded signals from the AMMC neurons.

In response to broadband stimuli, we found that JON population responses decrease in magnitude with increasing stimulus intensity. The JONs are mechanically coupled to the arista, which vibrates in response to sound stimulation. A previous study found that arista responses, assayed with laser Doppler vibrometry, shift their peak response frequency with increasing intensity of white-noise stimulation, across a similar intensity range (Göpfert and Robert, 2002). While we do not know how JON frequency responses might shift for different white-noise intensities and angles of the incident sound wave than those we examined (see Materials and Methods), we hypothesize that intrinsic cellular properties of the JONs may transform response curves of the arista, to maximize responses at certain distances from the sound source (and therefore particular intensities) and diminish responses to higher intensities (when the male fly comes too close to the female's receiver, for example), across a broad frequency range. Indeed, other studies that have assayed the JON population with LFP recordings have found that for high intensity tonal stimuli $(>1 \mathrm{~mm} / \mathrm{s})$, responses decrease with increasing intensity (Kamikouchi et al., 2009; Effertz et al., 2011). As a future direction, it would be useful to characterize for our delivery system, designed to mimic courtship conditions, sound responses using simultaneous laser Doppler vibrometry and electrophysiology over a larger range of stimulus intensities and angles than we have examined. Finally, identifying the array of ion channels expressed in the JONs should shed light on biophysical mechanisms that may shift tuning curves between the arista and the JO.

Our study reveals a complex transformation in response profiles between the summed output of the JON population and AMMC-A1 neurons in the brain, at steady state. While we do not know which of the $\sim 50-100$ JO-A neurons are presynaptic to AMMC-A1 neurons, and therefore cannot compare tuning directly between presynaptic and postsynaptic partners, we do not observe a similarly complex transformation for responses to pulse stimuli. One possibility to explain this difference between steady-state and transient tuning is that the JON LFP represents the activity of JO-A neurons for transients, but not at steady state (that is, other JO neurons that innervate different zones of the AMMC dominate the response at steady state). We think this possibility is unlikely given previous calcium imaging experiments, which demonstrated that JO-A+B neurons produce the strongest steady-state responses to sinusoidal vibration (com- 
pared with JO-CE neurons), and of the A+B neurons, JO-A neurons respond preferentially to frequencies between 100 and 900 $\mathrm{Hz}$ (Kamikouchi et al., 2009). This same study reported that killing either the JO-B or JO-CE neurons did not affect JO steadystate intensity tuning profiles (in response to sinusoidal stimuli), but that killing JO-A neurons specifically shifted tuning curves to higher intensities. While the strength of enhancer lines used for neuronal killing may have varied across these experiments, these data collectively suggest that JO-A neurons dominate the JO response to vibratory stimuli in the frequency and intensity range we examined. We therefore posit that the transformation at steady state arises rather from an active mechanism within the AMMC neurons themselves. Such a mechanism may involve synaptic adaptation during responses to longer stimuli, which are characterized by sustained depolarization of the membrane potential. Indeed, for responses to pulse trains composed of short IPIs (20 ms), we observed larger DC shifts in the membrane potential than for longer IPIs, but the same peak response amplitudes (Fig. 5G). This is consistent with data from the avian auditory system, in which a mixture of short-term facilitation and depression has been shown to similarly maintain subthreshold response amplitudes at even high stimulus rates (MacLeod et al., 2007). An alternative mechanism may involve a change in brain state during listening to natural sounds specifically (Engel et al., 2001); that is, a feedback system whose activity is based on the social importance of the sound might shape AMMC responses to match JO tuning curves for pulse songs specifically. Regardless of mechanism, differences in steady-state versus transient responses may have evolved based on the different functions of the mechanosensory system in flies. In response to sustained JON activity at steady state (during constant low-amplitude air flow, for example), the tuning curves of the AMMC-A1 neurons allow the system to respond preferentially to low stimulus frequencies, while remaining more invariant to changes in intensity. However, in response to onsets or song pulses, both ethologically relevant stimuli, AMMC-A1 neurons follow the tuning of the JON population, to reliably pass on information about stimulus timings and intensities, to downstream circuits for analysis. Such differences in steady-state versus transient responses have been observed in other systems (Mechler et al., 1998), and may be important for encoding natural stimuli, which, regardless of sensory modality, largely consist of transients (Rieke et al., 1997).

We have characterized detailed tuning curves for one subset of neurons within the AMMC. We do not know, however, how these tuning curves might differ for other AMMC neurons, nor which AMMC neurons are involved in the female's song response. We can therefore only correlate the responses we record with potential behavioral significance. In light of this, do our recordings offer any insights into mechanisms for speciesspecific song recognition? Pulse song responses are similar at the level of the JO in D. mel and D. sim, and are likely also to be similar at the level of the AMMC-A1 neurons in these two species. These results may hint at the fact that the detection of conspecific song happens downstream from the AMMC. However, measurable DC shifts (sustained depolarization) in the AMMC-A1 membrane potential occur in response to pulse songs with IPIs $\leq 35$ ms (estimated using the same simulation described in Fig. 6, but instead drawing from the measured AMMC-A1 decay times while systematically varying IPI). If this elevation of the membrane potential is sufficient to support neurotransmitter release (e.g., by causing sustained $\mathrm{Ca}^{2+}$ entry into the presynaptic terminal of the AMMC neurons), it could signal the preferred IPI. However, according to this hypothesis, the D. mel IPI range [30 \pm
$5 \mathrm{~ms}$ (Cowling and Burnet, 1981)] would also produce a DC shift in D. sim AMMC-A1 neurons, which likely have similar decay constants (Fig. 6). This might not pose a problem to $D$. sim females, as, due to large differences in pheromone profiles, $D$. mel males rarely, if ever, sing to them (Moulin et al., 2004). That is, decay times may not have diverged in $D$. sim due to a lack of evolutionary pressure to do so. The broad range of $D$. sim IPIs overlaps that of $D$. mel; therefore, $D$. sim males, which court $D$. mel females, occasionally produce IPIs that can drive DC shifts in D. mel AMMC neurons. D. mel females could then use this DC shift (driven more reliably by $D$. mel song) to signal the difference between the two species. Our model could be validated by comparisons in other species pairs that hybridize in the wild, but with different pulse songs, such as Drosophila yakuba and Drosophila santomea or Drosophila pseudoobscura and Drosophila persimilis (Barbash, 2010), and the development of genetic tools in other Drosophila species should permit such experiments (Holtzman et al., 2010). The mechanism we propose suggests that early auditory neurons (first- and second-order neurons) already contain sufficient information for making distinctions between species; there is precedent for this in both the grasshopper (Machens et al., 2003) and cricket (Nabatiyan et al., 2003) auditory systems.

In summary, we find that responses to fly song are well matched between the JON population and AMMC-A1 neurons, and that passing the JO population response through a simple linear filter recapitulates the AMMC-A1 response to song. AMMC-A1 neurons thus filter out information about pulse frequency that is encoded by the JONs (in the frequency doubling the JONs produce at the population level). Put another way, the JONs set the range of frequencies and intensities that the auditory system can respond to, and AMMC neurons, one synapse downstream from the JONs, filter the JON input to primarily preserve information about pulse timings, which are known to have behavioral relevance during courtship (Bennet-Clark and Ewing, 1969). The fly auditory system may thus turn out to be an excellent model for studying the neural encoding of temporal patterns and sequences. By characterizing tuning in fine detail for one neuron type within the AMMC, our study sheds a first light on how sound is processed along the early stages Drosophila auditory pathway. Future studies should be directed at determining (1) if all AMMC neurons are similarly tuned and, if so, what synaptic properties between JONs and AMMC neurons establish these tuning curves, and (2) the identities and response profiles of downstream neurons in the Drosophila auditory pathway that read out these auditory codes from the AMMC.

\section{References}

Albert JT, Nadrowski B, Göpfert MC (2007) Mechanical signatures of transducer gating in the Drosophila ear. Curr Biol 17:1000-1006.

Alle H, Geiger JR (2006) Combined analog and action potential coding in hippocampal mossy fibers. Science 311:1290-1293.

Barbash DA (2010) Ninety years of Drosophila melanogaster hybrids. Genetics 186:1-8.

Bar-Yosef O, Rotman Y, Nelken I (2002) Responses of neurons in cat primary auditory cortex to bird chirps: effects of temporal and spectral context. J Neurosci 22:8619-8632.

Bass AH, McKibben JR (2003) Neural mechanisms and behaviors for acoustic communication in teleost fish. Progr Neurobiol 69:1-26.

Bennet-Clark HC (1971) Acoustics of insect song. Nature 234:255-259.

Bennet-Clark HC, Ewing AW (1969) Pulse interval as a critical parameter in the courtship song of Drosophila melanogaster. Anim Behav 17:755-759.

Bentley DR, Hoy RR (1972) Genetic control of the neuronal network generating cricket (Teleogryllus gryllus) song patterns. Anim Behav 20:478-492.

Braaten RF, Reynolds K (1999) Auditory preference for conspecific song in isolation-reared zebra finches. Anim Behav 58:105-111. 
Burrows M, Siegler MV (1978) Graded synaptic transmission between local interneurones and motor neurones in the metathoracic ganglion of the locust. J Physiol 285:231-255.

Büschges A (1995) Role of local nonspiking interneurons in the generation of rhythmic motor activity in the stick insect. J Neurobiol 27:488-512.

Clark AG, Eisen MB, Smith DR, Bergman CM, Oliver B, Markow TA, Kaufman TC, Kellis M, Gelbart W, Iyer VN, Pollard DA, Sackton TB, Larracuente AM, Singh ND, Abad JP, Abt DN, Adryan B, Aguade M, Akashi H, Anderson WW, et al. (2007) Evolution of genes and genomes on the Drosophila phylogeny. Nature 450:203-218.

Cowling DE, Burnet B (1981) Courtship songs and genetic control of their acoustic characteristics in sibling species of the Drosophila melanogaster subgroup. Anim Behav 29:924-935.

David SV, Vinje WE, Gallant JL (2004) Natural stimulus statistics alter the receptive field structure of V1 neurons. J Neurosci 24:6991-7006.

Dermitzakis ET, Masly JP, Waldrip HM, Clark AG (2000) Non-mendelian segregation of sex chromosomes in heterospecific Drosophila males. Genetics 154:687-694.

Eberl DF, Hardy RW, Kernan MJ (2000) Genetically similar transduction mechanisms for touch and hearing in Drosophila. J Neurosci 20:5981-5988.

Effertz T, Wiek R, Göpfert MC (2011) NompC TRP channel is essential for Drosophila sound receptor function. Curr Biol 21:592-597.

Engel AK, Fries P, Singer W (2001) Dynamic predictions: oscillations and synchrony in top-down processing. Nat Rev Neurosci 2:704-716.

Engel JE, Wu CF (1996) Altered habituation of an identified escape circuit in Drosophila memory mutants. J Neurosci 16:3486-3499.

Fotowat H, Fayyazuddin A, Bellen HJ, Gabbiani F (2009) A novel neuronal pathway for visually guided escape in Drosophila melanogaster. J Neurophysiol 102:875-885.

Göpfert MC, Robert D (2001) Biomechanics. Turning the key on Drosophila audition. Nature 411:908.

Göpfert MC, Robert D (2002) The mechanical basis of Drosophila audition. J Exp Biol 205:1199-1208.

Göpfert MC, Robert D (2003) Motion generation by Drosophila mechanosensory neurons. Proc Natl Acad Sci U S A 100:5514-5519.

Göpfert MC, Albert JT, Nadrowski B, Kamikouchi A (2006) Specification of auditory sensitivity by Drosophila TRP channels. Nat Neurosci 9:999-1000.

Heil P (1997a) Auditory cortical onset responses revisited. I. First-spike timing. J Neurophysiol 77:2616-2641.

Heil P (1997b) Auditory cortical onset responses revisited. II. Response strength. J Neurophysiol 77:2642-2660.

Holtzman S, Miller D, Eisman R, Kuwayama H, Niimi T, Kaufman T (2010) Transgenic tools for members of the genus Drosophila with sequenced genomes. Fly 4:349-362.

Hsu A, Woolley SM, Fremouw TE, Theunissen FE (2004) Modulation power and phase spectrum of natural sounds enhance neural encoding performed by single auditory neurons. J Neurosci 24:9201-9211.

Joesch M, Plett J, Borst A, Reiff DF (2008) Response properties of motionsensitive visual interneurons in the lobula plate of Drosophila melanogaster. Curr Biol 18:368-374.

Kamikouchi A, Shimada T, Ito K (2006) Comprehensive classification of the auditory sensory projections in the brain of the fruit fly Drosophila melanogaster. J Comp Neurol 499:317-356.

Kamikouchi A, Inagaki HK, Effertz T, Hendrich O, Fiala A, Göpfert MC, Ito K (2009) The neural basis of Drosophila gravity-sensing and hearing. Nature 458:165-171.

Kelley DB (2004) Vocal communication in frogs. Curr Opin Neurobiol 14:751-757.

Kretzberg J, Warzecha AK, Egelhaaf M (2001) Neural coding with graded membrane potential changes and spikes. J Comput Neurosci 11:153-164.

Laurent G (1991) Evidence for voltage-activated outward currents in the neuropilar membrane of locust nonspiking local interneurons. J Neurosci 11:1713-1726.

Machens CK, Schütze H, Franz A, Kolesnikova O, Stemmler MB, Ronacher B, Herz AV (2003) Single auditory neurons rapidly discriminate conspecific communication signals. Nat Neurosci 6:341-342.

Machens CK, Gollisch T, Kolesnikova O, Herz AV (2005) Testing the efficiency of sensory coding with optimal stimulus ensembles. Neuron $47: 447-456$
MacLeod KM, Horiuchi TK, Carr CE (2007) A role for short-term synaptic facilitation and depression in the processing of intensity information in the auditory brain stem. J Neurophysiol 97:2863-2874.

Mason AC, Faure PA (2004) The physiology of insect auditory afferents. Microsc Res Tech 63:338-350.

Mechler F, Victor JD, Purpura KP, Shapley R (1998) Robust temporal coding of contrast by V1 neurons for transient but not for steady-state stimuli. J Neurosci 18:6583-6598.

Moulin B, Aubin T, Jallon JM (2004) Why there is a one-way crossability between $D$. melanogaster and D. simulans? An ontogenic explanation. Genetica 120:285-292.

Murthy M, Turner GC (2010) In vivo whole-cell recordings in the Drosophila brain. In: Drosophila neurobiology: a laboratory manual (Zhang B, Freeman M, Waddell S, eds). Cold Spring Harbor, NY: Cold Spring Harbor Laboratory.

Murthy M, Fiete I, Laurent G (2008) Testing odor response stereotypy in the Drosophila mushroom body. Neuron 59:1009-1023.

Nabatiyan A, Poulet JF, de Polavieja GG, Hedwig B (2003) Temporal pattern recognition based on instantaneous spike rate coding in a simple auditory system. J Neurophysiol 90:2484-2493.

Nadrowski B, Albert JT, Göpfert MC (2008) Transducer-based force generation explains active process in Drosophila hearing. Curr Biol 18:1365-1372.

Nadrowski B, Effertz T, Senthilan PR, Göpfert MC (2011) Antennal hearing in insects-new findings, new questions. Hear Res 273:7-13.

Petkov CI, Kayser C, Steudel T, Whittingstall K, Augath M, Logothetis NK (2008) A voice region in the monkey brain. Nat Neurosci 11:367-374.

Phelan P, Goulding LA, Tam JL, Allen MJ, Dawber RJ, Davies JA, Bacon JP (2008) Molecular mechanism of rectification at identified electrical synapses in the Drosophila giant fiber system. Curr Biol 18:1955-1960.

Riabinina O, Dai M, Duke T, Albert JT (2011) Active process mediates species-specific tuning of Drosophila ears. Curr Biol 21:658-664.

Rieke F, Bodnar DA, Bialek W (1995) Naturalistic stimuli increase the rate and efficiency of information transmission by primary auditory afferents. Proc Biol Sci 262:259-265.

Rieke F, Warland D, de Ruyter van Steveninck RR, Bialek W (1997) Spikes: exploring the neural code. Cambridge, MA: MIT.

Rotman Y, Bar-Yosef O, Nelken I (2001) Relating cluster and population responses to natural sounds and tonal stimuli in cat primary auditory cortex. Hear Res 152:110-127.

Shu Y, Hasenstaub A, Duque A, Yu Y, McCormick DA (2006) Modulation of intracortical synaptic potentials by presynaptic somatic membrane potential. Nature 441:761-765.

Smith EC, Lewicki MS (2006) Efficient auditory coding. Nature 439: 978-982.

Sturtevant AH (1920) Genetic studies on Drosophila simulans. I. Introduction. Hybrids with Drosophila melanogaster. Genetics 5:488-500.

Suga N (1989) Principles of auditory information-processing derived from neuroethology. J Exp Biol 146:277-286.

Theunissen FE, Shaevitz SS (2006) Auditory processing of vocal sounds in birds. Curr Opin Neurobiol 16:400-407.

Todi SV, Sharma Y, Eberl DF (2004) Anatomical and molecular design of the Drosophila antenna as a flagellar auditory organ. Microsc Res Tech 63:388-399.

von Schilcher F (1976) The role of auditory stimuli in the courtship of Drosophila melanogaster. Anim Behav 24:18-26.

Werner E (2008) Dynamic pressure difference microphones. In: Handbook of signal processing in acoustics, Vol I (Havelock D, Kuwano S, Vorländer M, eds), pp 1275-1282. Osaka: Springer.

Wightman FL, Kistler DJ (1989) Headphone simulation of free-field listening. I: Stimulus synthesis. J Acoust Soc Am 85:858-867.

Wilson RI, Turner GC, Laurent G (2004) Transformation of olfactory representations in the Drosophila antennal lobe. Science 303:366-370.

Woolley SM, Gill PR, Theunissen FE (2006) Stimulus-dependent auditory tuning results in synchronous population coding of vocalizations in the songbird midbrain. J Neurosci 26:2499-2512.

Yorozu S, Wong A, Fischer BJ, Dankert H, Kernan MJ, Kamikouchi A, Ito K, Anderson DJ (2009) Distinct sensory representations of wind and nearfield sound in the Drosophila brain. Nature 458:201-205. 